\title{
EDITORIAL
}

\section{Medio siglo de vida y progresos en la Escuela de Odontología de la Universidad del Valle, Cali - Colombia: hechos relevantes de la última década}

\section{Half Century of creation and progress at the Dental School - Universidad del Valle, Cali - Colombia: relevant achievements in the last decade}

\author{
Judy Villavicencio $^{1}$ (D) $\mid$ Adolfo Contreras ${ }^{2}$ (D) Jesús Hernández Silva $^{1}$ (D) $\mid$ Freddy Moreno $^{3}$ (D)
}

\section{ACCESO ABIERTO}

${ }^{1}$ Universidad del Valle, Facultad de Salud, Escuela de Odontología, Departamento de Odontopediatría y Ortopedia Maxilar, Cali, Colombia.

${ }^{2}$ Universidad del Valle, Facultad de Salud, Escuela de Odontología, Grupo de Medicina Periodontal, Cali, Colombia.

${ }^{3}$ Pontificia Universidad Javeriana, Facultad de Ciencias de la Salud, Grupo Ciencias ásicas y Clínicas de la Salud, Cali, Colombia.

Citación

Villavicencio J, Contreras A,

Hernández J, Moreno F. Medio siglo de vida y progresos en la Escuela de Odontología de la Universidad del Valle, Cali Colombia: hechos relevantes de la última década. Rev

Estomatol. 2019;27(2):1-10.

DOI: $10.25100 /$ re.v27i2.8169

Correspondencia Judy Villavicencio. Universidad del Valle. Calle 4b \# 36b-00 Edificio 132. Email: judy.villavicencio@correouniva lle.edu.co
Hace cincuenta años, el 4 de junio de 1970, fue creado el Departamento de Estomatología adscrito a la División de Salud, el cual dio origen en 1990 a la Escuela de Odontología, que estará celebrando medio siglo de creación y desarrollo en el 2020 (1).

Inicialmente, el 27 de febrero de 1957, se había nombrado un comité para estudiar la creación de la Facultad de Odontología en la Universidad del Valle. Dicho comité incluyó, entre otros, a los médicos Luis María Borrero y Pablo Barreto Reyes, y a los odontólogos Itamar Alcalay, Gustavo Ulloa Iragorry y Jaime Ángel. Sin embargo, aunque la propuesta presentada en el Primer Plan Quinquenal de Desarrollo de la Facultad de Medicina -auspiciada por las Fundaciones Rockefeller y Kellogg- permitió el fortalecimiento y expansión de los Departamentos de Ciencias Básicas Médicas de la Facultad, este no contó con la aprobación para crear un programa de odontología, debido al alto costo de la propuesta, asociado a la dotación de infraestructura y equipos para los laboratorios, preclínicas y clínicas (2).

Este artículo pretende recopilar los más relevantes hitos de un largo camino que se inició oficialmente con la creación en 1965 del Comité Universitario Pro Facultad de Odontología CUPFO, integrado por los odontólogos José Fernando Barreto y Jaime Álvarez, entre otros. Dicho comité impulsó la creación del Departamento de Estomatología, el de junio de1970. Para entonces un selecto grupo de profesionales de la Odontología de Cali liderados por el doctor José Fernando Barreto le propusieron a la Organización Panamericana de la Salud-OPS y a los Ministerios de Salud y de Educación la creación de dos programas revolucionarios para la época, un pregrado en Odontología de cuatro años de duración, el cual al ser finalizado daba la posibilidad de continuar con estudios de Magíster en Estomatología con dos años adicionales de estudio; el servicio social obligatorio contaría como uno de los años de la Maestría.

Copyright:

(C) Universidad del Valle. 
Ambos programas de estudios estaban apoyados por trabajos previos en donde, se destacan los programas de auxiliares de Odontología, cuyo espectro contemplaba desde los niveles mas básicos del personal de apoyo a los servicios de odontología representadas en las auxiliares de consultorio, niveles intermedios de complejidad como el programa de auxiliares de higiene oral o higienista, y un programa novedoso para Colombia y América Latina como eran los auxiliares de Odontología social que tenia funciones de operatorista y que trabajarían con la supervisión del odontólogo y/o estomatólogo, y cuya función era incrementar el acceso al cuidado de la salud bucal, una necesidad real de los Colombianos para esa época. La auxiliar de Odontología Social, se derivaba del programa o enfermera dental en Nueva Zelanda (1).

Los principales principios del nuevo profesional de la Odontología eran: 1 . Tener un fuerte conocimiento y uso de las ciencias básicas biomédicas para fortalecer su trabajo clínico; 2. Poseer la capacidad de implementar y supervisar programas de estomatología social y/o comunitaria en los puestos, centros de salud y hospitales de la red publica, a nivel departamental, con enfoque de atención primaria de la salud y en donde lo primordial es intervenir los riesgos en salud del grupo familiar, con el apoyo de las auxiliares de Odontología, 3. Fortalecer la promoción de la salud, el uso de los equipos de odontología simplificado y el trabajo a cuatro manos con auxiliares de consultorio, auxiliares de higiene oral, y auxiliares de odontología social para masificar la promoción de la salud y la atención en salud entre la población, 4. Consolidar al estomatólogo (médico de la boca), como el líder de la pirámide del equipo de salud bucal con fortalezas en el diagnostico, prevención y tratamiento de las principales patologías bucales y su relación son la salud general, con capacidad resolutiva y las competencias para la dirección y coordinación de los equipos de salud para trabajar en el ámbito gubernamental, en los puestos de salud y centros hospitales del Departamento del Valle y en el área de influencia del sur-occidente Colombiano $(1,2)$.
Sin embargo, aquellos sueños totalmente novedosos para la época, fueron transformándose a lo largo del desarrollo del primer plan de estudios que se inicio en 1975 y las ideas originales se modificaron en el tiempo por las siguientes razones: 1. Los programas propuestos era demasiado revolucionarios y tenían un fuerte enfoque social que confrontaba con el ejercicio mas liberal y privado de la Odontología en esa época, 2. Los colegas que acompañaron la creación de los nuevos programas fueron criticados por la Federación Odontológica Colombiana, pues aquellos visualizaron la auxiliar de Odontología social como un recurso humano que competía con el odontólogo graduado, y agravaba problema del ejercicio empírico de la odontología, 3. El Ministerio de Salud y el sector gubernamental de la salud, no tenían la capacidad de emplear a las egresadas de los programas de higiene oral y de Odontología social y efectivamente algunas ejercieron, como empíricos, incrementando las críticas a los programas de auxiliares y la desconfianza en el gremio odontológico e incluso en algunos docentes de la Escuela, 4. el doctor José Fernando Barreto, líder de la propuesta de formación de personal auxiliar y del nuevo profesional de la Odontología, asumió otras funciones en la Facultad de Salud y en la Universidad del Valle y fue reemplazado por nuevos profesionales, mas tradicionales y conservadores en la visión del ejercicio de la odontología para ese tiempo, y por ultimo, 5. Los estudiantes de la primera promoción, al notar los cambios evidentes en el programa, y la falta de docentes en algunas áreas, iniciaron un paro que fue respaldado por el resto de los estudiantes, y hubo cambios forzados en la estructura del plan de estudios y fueron contratados nuevos docentes que poco conocían el plan inicial propuesto (1); por estas razones y otras que hacen parte del natural olvido con el paso de los años, estos sueños de tener un estomatólogo en la pirámide de la formación, continúan hoy siendo sueños inconclusos de un pasado que pudo ser mejor; sin embargo a pesar de estas dificultades, nuestros docentes y egresados llevan en el ADN trazas de ese pasado innovador y progresista que por diversas razones no logró consolidarse totalmente. 
La Escuela cuenta con dos publicaciones hechas al cumplir 30 y 40 años, en las que se recopiló la historia desde las actas, resoluciones, datos, anécdotas, testimonios, evolución, desarrollo y contribución realizada por cada una de las personas que han aportado a hacer de esta Institución de formación de talento humano en salud bucodental como una de las mas relevantes en el Suroccidente Colombiano y que invitamos a revisar a aquellos que deseen conocer mayores detalles del pasado $(1,2)$.

Con este documento pretendemos dejar plasmadas los aspectos mas significativos sucedidos en la Escuela de Odontología en los últimos 10 años (2009-2019), como parte de un homenaje a esta Unidad Académica que despierta orgullo y agradecimiento al permitirnos ser parte de ella como egresados, , empleados profesores y directivos.

En el año 2009 se inician las actividades de acompañamiento a la Estrategia de Salud Familiar de la Red de Salud de Ladera, en las Comunas 18 y 20 basada en la Atención Primaria en Salud y que cobijaba una población de 173.453 caleños (3). Cinco estudiantes del Programa Académico de Odontología estuvieron entre los mejores puntajes a nivel nacional en los Exámenes de Calidad de la Educación Superior (ECAES).

El profesor del Programa Académico de Odontología Freddy Moreno, gana el premio a la mejor investigación en el área de biomateriales dentales en el III Congreso de la Región Latinoamericana de la International Association of Dental Research (IADR), realizado en la Isla de Margarita, con el trabajo "Comportamiento de tejidos y materiales dentales sometidos a altas temperaturas". En el marco de los 64 años de la Universidad del Valle, el Consejo Superior otorgó la distinción académica de Profesor Emérito al docente Adolfo Contreras por su desempeño como investigador.

En el 2010 se formaliza la reforma del Programa Académico de Odontología, plasmada en la
Resolución 096 del 9 de Septiembre de 2010 del Consejo Académico (4), con esta se le da una mayor organización a los componentes, créditos y prerrequisitos del Programa. Tres estudiantes del Programa Académico de Odontología se destacaron por sus puntajes a nivel nacional en los ECAES. Se da inicio al proyecto "Alianza por un futuro libre de caries" como una iniciativa pionera en Latinoamérica; en Colombia, la Alianza está conformada por la Asociación Colombiana de Facultades de Odontología (ACFO), la Universidad Autónoma de Manizales (UAM), la Universidad el Bosque, la Universidad de Antioquia y la Universidad del Valle trabajando en equipo con las autoridades sanitarias, las IPS y otras organizaciones.

La Alianza por un Futuro Libre de Caries aporta al objetivo de tener comunidades más sanas, con mejores prácticas de autocuidado y con mejores alternativas de promoción de la salud buco dental; el proyecto global está constituido por líderes y dirigentes mundiales de la odontología y de la salud pública, academia, profesionales, gremios, gobierno e instituciones privadas para promover medidas de salud pública que permitan prevenir y controlar la caries dental, enfermedad de alta prevalencia que genera altos costos y deterioro de la calidad de vida de las personas; el Área Comunitaria del Programa Académico de Odontología ha centrado esta estrategia en las instituciones educativas y de salud de la Comuna 18 y 20 de Santiago de Cali, siendo la profesora Lina María García y Ana Fernanda Jaimes las principales gestoras de la Alianza por parte de la Escuela de Odontología.

El Consejo Superior de la Universidad del Valle otorgó la distinción académica de Profesor Distinguido al docente Jesús Alberto Hernández (5). La profesora Beatriz Guzmán asume el cargo de Jefe de la Sección del Servicio Odontológico, sede Meléndez, el cual hace parte del Sistema de Salud de la Universidad del Valle; este cargo lo ejerce a la fecha y la gestión desarrollada se ha caracterizado por la modernización del mismo y el énfasis a la 
comunidad universitaria sobre la promoción y la prevención de las enfermedades bucodentales.

2011. El profesor Jesús Alberto Hernández es reelegido Director de la Escuela de Odontología para el periodo 2011-2013. El Profesor Adolfo Contreras gana el "Premio Scopus Colombia 2011, galardón que reconoce la labor de los investigadores del país que se destacan a nivel internacional por el número de citaciones en Elsevier y Scopus. En el marco del XXXV Congreso Nacional de Cirugía Oral y Maxilofacial, la profesora Janneth Zúñiga obtuvo el primer puesto por la investigación "Cuantificación por cuadrantes de la distorsión presente en la radiografía panorámica convencional"; la profesora se desempeña en la Escuela de Ciencias Básicas de la Universidad del Valle.

La investigación "Microchips implantados en molares sometidos a fuerzas compresivas y altas temperaturas" liderada por el profesor Freddy Moreno, obtuvo el Premio de Investigación IADR Colombia Hatton - Unilever otorgado por la Asociación Internacional para la Investigación en Odontología IADR, División Colombia.

En el 2012 la profesora Libia Soto es designada directora de la Sede Regional de Tuluá, cargo que ejerce hasta la fecha habiéndose distinguido por su gestión financiera y contable basada en la administración efectiva con potencialización de los recursos de la Sede y por el incremento en la oferta académica. La profesora Judy Elena Villavicencio es nombrada como Vicedecana Académica de la Facultad de Salud 2012-2014, en este periodo su gestión se enfatizó en la adaptación y cumplimiento de todos los programas de la Facultad a los lineamientos del Decreto 2376 de 2010 del Ministerio de la Protección Social y el Ministerio de Educación Nacional (6), que regula la relación docencia servicio para los programas de formación de talento humano del área de la salud.

Las directivas de la Escuela de Odontología y de la Facultad de Salud, le brindaron un homenaje al fundador de la Escuela de Odontología Doctor José Fernando Barreto Reyes, en el marco del "Congreso 4
Internacional Escuela de Odontología 42 años de creación". La investigación "Genotipificación de Porphyromonas gingivalis Fim-A en pacientes con gingivitis y periodontitis en la ciudad de Cali Colombia" liderada por la profesora Sandra Milena Moreno, obtuvo el Premio de Investigación IADR Colombia Hatton - Unilever otorgado por la Asociación Internacional para la Investigación en Odontología IADR, División Colombia.

El profesor Adolfo Contreras asume el cargo de director del Centro para el Desarrollo y Evaluación de Políticas y Tecnología en Salud Pública (CEDETES), cargo que ostentó hasta mediados del 2016 y donde se destacan los diez y seis (16) proyectos realizados, dos encuestas poblacionales para prevenir las enfermedades crónicas no trasmisibles en Cali en convenio con la Alcaldía de Cali, una asesoría técnica a la subdirección de enfermedades crónicas no trasmisibles del Ministerio de Salud y Protección Social, y el proyecto de promoción, educación y comunicación para la salud con enfoque diferencial para la Organización Panamericana de la Salud.

En el 2013 se emitió concepto favorable de las Clínicas de la Escuela de Odontología como escenario de práctica de docencia servicio, por parte de la Comisión Intersectorial para el Talento Humano en Salud, en consonancia con el Decreto 2376 de 2010 (6). Se da la renovación del registro calificado del Programa de Especialización en Periodoncia, mediante la Resolución No. 16724 del 20 de noviembre de 2013 del Ministerio de Educación Nacional (7); este proceso es liderado por el coordinador del programa profesor Jorge Enrique Soto. La Escuela de Odontología se acoge a la Norma General de Archivo de las entidades públicas Colombianas. Se obtuvo un primer puesto en el XXI Congreso Internacional de Ortodoncia con el trabajo "Efectos clínicos y sistémicos de la ortodoncia osteogénica acelerada periódicamente" y en el Golden Bracket Award por el trabajo "Colonización bacteriana en brackets estéticos de autoligado, estudio in vitro", estas investigaciones fueron lideradas por los profesores Wilhelm Bellaiza, Mario Ortiz y Juan Fernando Aristizabal. 


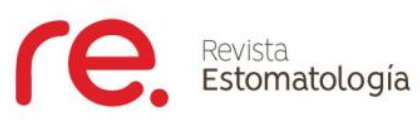

En el 2014 el profesor Alberto Calero inició la gestión como director de la Escuela de Odontología periodo 2014-2016. Ese año el Consejo Nacional de Acreditación otorgó, mediante la Resolución No. 12894 del 11 de agosto de 2014 (8), la renovación por ocho años de la Acreditación de Alta Calidad al Programa Académico de Odontología; destacó del Programa el plan de estudios que permite formar profesionales integrales, comprometidos con la salud de la comunidad, fundamentado en un análisis permanente de los retos de la profesión y su entorno académico, social y cultural; la producción académica de los profesores publicada en revistas indexadas de circulación nacional e internacional, sumado a materiales de apoyo docente; el buen desempeño de los estudiantes en las pruebas SaberPro durante los años de la renovación de la acreditación, obteniendo puestos destacados.

El Ministerio de Educación Nacional avaló el inicio de actividades del Programa de Especialización en Endodoncia mediante la Resolución No. 13092 del 13 de agosto de 2014 (9); esta iniciativa fue liderada por la profesora Patricia Rodríguez. El Consejo Académico convoca al concurso público de méritos, selección y vinculación de los candidatos a profesores de tiempo completo en el marco del Programa de Semilleros Docentes; esta Convocatoria garantizó la formación doctoral para un profesor de la Escuela de Odontología en ciencias básicas odontológicas y otro en ciencias clínicas odontológicas (10). El proyecto de investigación "Efectos de un alimento enriquecido con probióticos a base de Lactobacillus rhamnosus GG sobre el recuento de Streptoccocus mutans y Lactobacillus $\mathrm{sp}$ y la presencia de caries incipiente en niños de 3 a 4 años en Cali, Colombia", recibe financiación para su desarrollo por parte de Colciencias y la Universidad del Valle, este ensayo clínico controlado aleatorizado pretendía evaluar los cambios en el recuento microbiológico, el índice de placa dental y características físico-químicas de la saliva, posterior a la administración de un alimento enriquecido con bacterias probióticas. Se da la renovación del registro calificado del Programa de Especialización en Ortodoncia, mediante la Resolución No. 22666 del 29 de diciembre de 2014 (11); este proceso es liderado por el coordinador del programa profesor Juan Fernando Aristizábal.

En el 2015 el Ministerio de Educación Nacional avala el inicio de actividades del Programa de Maestría en Ciencias Odontológicas con énfasis en investigación, mediante la Resolución No. 16121 del 30 de septiembre de 2015 (12); esta iniciativa se desarrolló a la par con la Facultades de Odontología de la Universidad de Antioquia, la Universidad Santo Tomás, y el liderazgo de la Universidad de Chile una de las universidades de más alto ranking en Latinoamérica. En el marco de los 70 años de la Universidad del Valle, el Consejo Superior estableció en la Resolución No. 004 de 2015 las distinciones académicas para los profesores de la Universidad del Valle (13); en esta ocasión fueron adjudicados al profesor Carlos Alfonso Mejía como Maestro Universitario, Gerardo Umaña como Profesor Emérito y Judy Villavicencio como Profesora Distinguida. El profesor Jairo Corchuelo asume la dirección del Hospital Universitario del Valle Evaristo García ESE, en momentos de la crisis más dura del centro asistencial en 60 años de existencia.

En el 2016 se lleva a cabo la renovación del registro calificado del Programa de Especialización en Odontología Pediátrica y Ortopedia Maxilar, mediante la Resolución No. 01222 del 28 de enero de 2016 (14). Así mismo se da la renovación del registro calificado del Programa de Especialización en Rehabilitación Oral, mediante la Resolución No. 09845 del 18 de mayo de 2016 (15); proceso dirigido por el coordinador del programa, profesor Herney Garzón. Se produce la renovación del registro calificado del Programa Académico de Odontología, mediante la Resolución No. 21946 del 22 de noviembre de 2016 (16). 
El coordinador del Programa de Especialización en Ortodoncia, Juan Fernando Aristizábal y algunos de sus residentes, participaron en el proyecto de investigación interinstitucional con la Universidad CES a la cabeza y con la Universidad de Michigan, titulado "Una estrategia de mejora del biotipo para el paciente sometido a ortodoncia acelerada", un ensayo clínico controlado aleatorizado, este pretendía evaluar y comparar la presencia o no de reabsorción radicular en un grupo de pacientes sometidos a tratamiento de ortodoncia con técnicas de corticotomía y refuerzo de colágeno con Mucograft ${ }^{\circledR}$, la financiación del proyecto se dio por la Osteology Foundation con sede en Suiza.

Este año el profesor Adolfo Contreras publica el artículo mas citado de la década 1999-2019; este es "La promoción de la salud general y la salud oral: una estrategia conjunta", publicado en la Revista Clínica de Periodoncia, Implantología y Rehabilitación Humana (Revista Q1), el cual ha sido citado por 47 autores y describe una estrategia poblacional para combinar la adopción de estilos de vida saludable para el control de las enfermedades crónicas no transmisibles y de las enfermedades orales, usando como pilares la estrategia de promoción, prevención y la educación a nivel individual y poblacional de los individuos sanos. Esta estrategia se contrapone con los actuales modelos curativos que demandan alta inversión y recursos físicos-humanos para el tratamiento de la enfermedad crónica y de las enfermedades orales. El profesor Contreras cuenta también con el artículo más citado en la historia de la Escuela de Odontología, el cual data del año de 1999.

En el 2017 el profesor Jesús Alberto Hernández inicia un nuevo periodo como director de la Escuela de Odontología. Gesta y participa en el Diplomado Internacional "Tratamiento temprano de las mal oclusiones en unión con la Sociedad Ecuatoriana de Ortodoncia de Ecuador. Se celebran los 20 años de creación del Programa de Especialización en Odontología Pediátrica y Ortopedia Maxilar.

Por primera vez se realizó la Ceremonia de Imposición de Batas para los estudiantes de quinto semestre del Programa Académico de Odontología; 6 esta simboliza la culminación del ciclo básico y la iniciación de las prácticas clínicas de los estudiantes con compromiso, respeto y seriedad para con sus pacientes. Las prácticas de ruralito reinician las actividades por parte de los estudiantes de último año del Programa Académico de Odontología en las sedes regionales de Tuluá y Zarzal; las directivas hacen un esfuerzo para optimizar las condiciones logísticas y locativas para las practicas de los estudiantes. Se da la publicación de los resultados de la encuesta SABE (salud, bienestar, y envejecimiento), la cual muestra los resultados de la situación de los adultos mayores en Colombia; para la realización de la Encuesta se constituyó una unión temporal entre la Universidad de Caldas y la Universidad del Valle, siendo el profesor Jairo Corchuelo el director de esta encuesta.

En el año 2018 y por primera en 66 años los profesores de la Facultad de Salud, eligen un decano no médico; honor que recae en el director de la Escuela de Odontología Jesús Alberto Hernández, su gestión se ha caracterizado por el apoyo al plan estratégico de desarrollo de cada una de la Escuelas que conforman la Facultad de Salud; por lo tanto se designa a la profesora Judy Villavicencio como directora de la Escuela de Odontología por un periodo de tres años.

El profesor Jairo Corchuelo se posesiona como director del Sistema de Regionalización de la Universidad del Valle; entre sus mayores logros se cuentan el haber concretado la convocatoria de contratación de 35 profesores a termino indefinido, la entrada en funcionamiento de la nueva sede en el campus las Balsas en la sede Zarzal, el apoyo a la creación por parte del Ministerio de Educación Nacional de la Maestría en Salud Ocupacional, Maestría en Políticas Públicas, Maestría en Educación y la Especialización en Calidad de la Gestión y Productividad.

El Programa Auxiliar en Salud Oral, pionero en formación de personal auxiliar en el suroccidente colombiano desde 1970, realiza una reforma curricular siendo uno de los hechos destacados el acondicionamiento del Programa por módulos y el 
paso de duración del Programa de tres semestres a dos semestres, en consonancia con los programas técnicos laborales de la región; esta iniciativa fue coordinada por la profesora Ana Piedad Delgado. En las instalaciones de la Facultad de Salud se desarrolló el VI Encuentro Regional de Investigación Odontológica de la Asociación Colombiana de Facultades de Odontología y el I Encuentro de semilleros de investigación ACFO, Nodo Suroccidente. Se llevó a cabo la campaña conjunta con la Facultad de Odontología de la Universidad Antonio Nariño, UNICOC-Cali y Universidad del Valle denominada "Sácale la lengua al cáncer", iniciativa que fue coordinada por la profesora Dora Ordoñez, en ella se realizó la valoración y la biopsia a pacientes con sospecha de una lesión pre maligna o maligna.

La Revista Estomatología aumenta su visibilidad ingresando a repositorios de revistas científicas como Redib, Lilacs, Google Scholar, BRS, Biblioteca virtual en Saude, Latindex, ICMJE, International Committee of Medical Journal Editors, DOAJ, Directory of Open Access Journals, EBSCO, GALE, Sherpa/Romeo, el profesor Edison Andrés Cruz Olivo ha sido el gestor de estas inclusiones. En la tercera versión de la Noche de Gala para Egresados las directivas de la Universidad del Valle hacen un reconocimiento a la egresada del Programa Académico de Odontología Erika Benavides, por la trayectoria profesional, que ha dejado en alto el nombre del Alma Mater; ella es profesora clínica asociada al Departamento de Periodoncia y Medicina Oral de la Facultad de Odontología de la Universidad de Michigan y diplomada y vicepresidenta de la Junta Americana de Radiología Oral y Maxilofacial (ABOMR) y se ha desempeñado como Concejal de Comunicaciones y Presidenta del Comité de Investigación y Tecnología de la Academia Americana de Radiología Oral y Maxilofacial (AAOMR). El Programa de Especialización en Periodoncia celebró 25 años de creación.
Para el 2019 se llevó a cabo la graduación de los estudiantes de la primera promoción de la Maestría en Ciencias Odontológicas, programa coordinado por el profesor Carlos Humberto Valencia. Los cuatro trabajos de investigación de esta primera cohorte fueron tesis meritorias. La modificación de la estructura curricular del Programa Académico de Odontología fue aprobada mediante Resolución No. 038 del 28 de febrero de 2019 del Consejo Académico de la Universidad del Valle (17); esta desencadenó en la modificación del registro calificado del Programa Académico de Odontología por el Ministerio de Educación Nacional de Colombia mediante la Resolución No. 8954 del 27 de Agosto de 2019 (18) y busca formar odontólogos con fundamentación en biología, investigación y ciencias sociales, con valores éticos y ciudadanos y competencias para promover la salud y prevenir las enfermedades bucodentales, diagnosticarlas y tratarlas las a nivel del individuo, la familia y la comunidad; esta iniciativa fue liderada por el profesor Adolfo Contreras, director del Programa Académico de Odontología (2018-2020).

La Escuela realizó el "Congreso 49 años de la Escuela de Odontología", aula Dentaid, en homenaje al profesor Jaime Álvarez Jaramillo, por su 42 años de servicio a la Institución en el Área de Cirugía Oral y Maxilofacial del cual fue su creador, artífice y pionero. Se dio la aprobación de tres patentes de invención por parte de la Superintendencia de Industria y Comercio de Colombia, expedidas mediante la Resolución No. 31716 al "Dispositivo de regeneración de hueso" (19); Resolución No. 60312, al "Kit de cemento óseo acrílico que comprende quitosano, óxido de grafeno, apatita, ácido poliláctico, cloruro de estroncio y comonómeros alcalinos" (20) y Resolución No. 60290 a la invención "Distractor histiogénico implanto soportado y protésicamente guiado" (21); el principal propulsor de estas iniciativas fue el profesor Carlos Humberto Valencia. 
El profesor Julián Balanta obtuvo un premio como joven investigador 2019, en Indiana Estados Unidos, en el marco del Encuentro de Investigación de interacciones hueso y músculo: la mecánica y más allá. El Programa de Especialización en Cirugía Oral y Maxilofacial es aprobado y creado por el Consejo Académico y el Consejo Superior de la Universidad del Valle, mediante las Resoluciones $(22,23)$; este proyecto fue liderado por la profesora Martha Rodríguez y continuará el transito por el Ministerio de Educación y el Ministerio de Salud y Protección Social para el inicio de actividades.

Estos logros de la última década y los años anteriores de la Escuela de Odontología de la Universidad del Valle, le han permitido graduar a 1075 odontólogos, 240 especialistas en Periodoncia, Odontología Pediátrica y Ortopedia Maxilar, Ortodoncia, Rehabilitación Oral, Endodoncia y 4 magister en Ciencias Odontológicas; todos ellos hoy en día desarrollan labores propias de la profesión, creando empresa, haciendo parte de instituciones educativas, instituciones de salud, de organizaciones relacionadas con la Odontología.

El desempeño de los egresados de la Escuela de Odontología se ha llevado a cabo principalmente en Cali y en Valle del Cauca, sin embargo también se tienen egresados ejerciendo en otros países como Italia, España, Finlandia, Alemania, Noruega, Australia, Corea de Sur, Chile, Ecuador, Brasil, Canadá, Estados Unidos; algunos son docentes en prestigiosas universidades como la Universidad de Michigan, Universidad de Augusta, la Universidad de la Florida, Universidad de Antioquia, Universidad Javeriana de Cali, Universidad Cooperativa de Colombia, Universidad Santiago de Cali, UNICOCCali.

Podemos concluir que la Escuela de Odontología en los últimos años ha contado con docentes y egresados que han tenido altos cargos académicoadministrativos en la Universidad del Valle y en otras instituciones educativas del país; gerentes de encuestas poblacionales como SABE y ENSE, profesores cuya investigación ha generado nuevos paradigmas de conocimiento a nivel latinoamericano 8 y mundial en temas como la etiopatogenesis de la enfermedad periodontal, el control de la caries de infancia temprana, la educación en salud bucodental, la aceleración de los tratamientos de ortodoncia, la odontología forense, las patentes de invención, también se cuenta con egresados desempeñándose en el sector salud como gerentes de EPS y de IPS públicas y privadas.

Avizoramos en el escenario la concreción de una reforma curricular en el Programa Académico de Odontología que pretende dar una mayor autonomía y mayores competencias en formación general (social y ciudadano, lenguaje y comunicación, estilos de vida saludable, artístico humanístico) a los futuros odontólogos, continuando claro está con las fortalezas en lo disciplinar.

La Escuela Odontología tiene grandes retos de cara al futuro, como son: 1. El seguir gestionando la concreción de las nuevas clínicas para la atención de pacientes, que estarían situadas en el "Edificio Multipropósito de la Facultad de Salud", proyectado en el lote contiguo de donde hoy se encuentra la Escuela; esta iniciativa cumpliría con las condiciones de habilitación reglamentadas por el Ministerio de Salud y Protección Social. 2. La concreción de la sistematización de las historias clínicas con anexos y ayudas diagnósticas con tecnología digital, que faciliten el tránsito entre las diferentes áreas de la Odontología, la sistematización de los procesos administrativos, financieros, contables y logísticos de la Escuela, este es otro gran reto para alcanzar el cual hasta ahora ha tenido logros parciales. 3. El compromiso de los programas académicos de pregrado y posgrado con procesos de acreditación de alta calidad tanto nacionales como internacionales, pretendiendo con ello ratificar la responsabilidad con el mejoramiento continuo y con el cumplimiento de estándares exigidos en un contexto global. 4. Ser un pilar fundamental en el desarrollo, actualización y evaluación de las decisiones en salud bucodental a nivel local y nacional, mediante la consolidación de programas académicos que cuenten con un centro de evidencia en odontología, y capacite a través del mismo a su comunidad. 5. La generación de nuevo conocimiento con impacto transversal, mediante la 
integración de la Odontología con otras áreas del conocimiento como la medicina, antropología, la zoología, la biología entre otras. 6. La concreción de trabajos conjuntos con la Facultad de Ingenierías y otras, que nos permita conformar una unidad robótica odontológica, donde se desarrollen prototipos de robots que puedan llevar a cabo tratamientos odontológicos de pacientes con enfermedades de alto riesgo de transmisibilidad.

\section{Referencias}

1. Moreno F, Moreno S, Martínez C, Ramírez F. Escuela de Odontología. 30 años. Federación Odontológica Estudiantil Universidad del Valle: Cali; 2000.

2. Sinisterra G, Barreto JF, Moreno F. Historia, evolución y desarrollo de la Escuela de Odontología de la Universidad del Valle. Santiago de Cali: Programa Editorial de la Universidad del Valle, 2010.

3. ALCALDIA DE SANTIAGO DE CALI. Cali en cifras 2009 [Internet]. Santiago de Cali: Impresora Feriva S.A; 2009. [citado en 11 de octubre de 2019]. Disponible en: https://planeacion.cali.gov.co/caliencifras/Documentos $\% 2$ 0pdf/Caliencifras2009.pdf

4. UNIVERSIDAD DEL VALLE. Resolución 096 del 9 de septiembre de 2010 "Por la cual se modifica y actualiza la Resolución 052 de febrero 26 de 2004".

5. UNIVERSIDAD DEL VALLE. Resolución 030 del 13 de mayo de 2010 del Consejo Superior "Por la cual se otorgan unas distinciones". [en línea] $<$ http://proxse16.univalle.edu.co/ secretariageneral/consej osuperior/resoluciones/2010/RCS-030.pdf> [citado en 16 de octubre de 2019]

6. MINISTERIO DE SALUD Y PROTECCIÓN SOCIAL Y MINISTERIO DE EDUCACIÓN NACIONAL. Acuerdo 00265 del 31 de agosto de 2016 "Por el cual se emite concepto técnico sobre la relación docencia servicio en el Programa de Odontología de la Universidad del Valle".

7. MINISTERIO DE EDUCACIÓN NACIONAL. Resolución 16724 del 20 de noviembre de 2034 "Por medio de la cual se resuelve la solicitud de renovación de registro calificado del programa de Especialización en Periodoncia de la Universidad del Valle para ser ofrecido bajo la metodología presencial en Cali - Valle del Cauca".

8. MINISTERIO DE EDUCACIÓN NACIONAL. Resolución 12894 del 11 de agosto de 2014 "Por medio del cual se renueva la acreditación de alta calidad al Programa Académico de Odontología por 8 años".

9. MINISTERIO DE EDUCACIÓN NACIONAL. Resolución 13092 del 13 de agosto de 2014 "Por medio de la cual se resuelve la solicitud de registro calificado del programa de
Especialización en Endodoncia de la Universidad del Valle para ser ofrecido bajo la metodología presencial en Cali Valle del Cauca".

10. UNIVERSIDAD DEL VALLE. Resolución 055 del 10 de abril de 2014 del Consejo Académico "Por la cual se convoca a concurso público de méritos, selección y vinculación de los candidatos a profesores de carrera de Tiempo Completo en el marco del Programa de Semilleros Docentes". [en línea] <http://proxse16.univalle.edu.co/ viceacademica//convoca toria_semillerodocente/2014//normatividad/res_055_ca.pdf $>$ [citado en 3 de septiembre de 2019].

11. MINISTERIO DE EDUCACIÓN NACIONAL. Resolución 22666 del 29 de diciembre de 2014 "Por medio de la cual se resuelve la solicitud de renovación de registro calificado del programa de Especialización en Ortodoncia de la Universidad del Valle para ser ofrecido bajo la metodología presencial en Cali - Valle del Cauca".

12. MINISTERIO DE EDUCACIÓN NACIONAL. Resolución 16121 del 30 de septiembre de 2015 "Por medio de la cual se resuelve la solicitud de registro calificado del programa de Maestría en Ciencias Odontológicas de la Universidad del Valle para ser ofrecido bajo la metodología presencial en Cali - Valle del Cauca".

13. UNIVERSIDAD DEL VALLE. Resolución 004 del 13 de febrero de 2015 del Consejo Superior "Por la cual se actualizan las distinciones académicas para los profesores de la Universidad del Valle y se reglamenta su otorgamiento". [en línea] $<$ http://proxse16.univalle.edu.co/ secretariageneral/consej o-superior/resoluciones/2015/RCS-004.pdf> [citado en 11 de octubre de 2019]

14. MINISTERIO DE EDUCACIÓN NACIONAL. Resolución 01222 del 27 de enero de 2016 "Por medio de la cual se resuelve la solicitud de renovación de registro calificado del programa de Especialización en Odontología Pediátrica y Ortopedia Maxilar de la Universidad del Valle para ser ofrecido bajo la metodología presencial en Cali - Valle del Cauca".

15. MINISTERIO DE EDUCACIÓN NACIONAL. Resolución 09845 del 18 de mayo de 2016 "Por medio de la cual se resuelve la solicitud de renovación de registro calificado del programa de Especialización en Rehabilitación Oral de la Universidad del Valle para ser ofrecido bajo la metodología presencial en Cali - Valle del Cauca".

16. MINISTERIO DE EDUCACIÓN NACIONAL. Resolución 21946 del 22 de noviembre de 2016 "Por medio de la cual se resuelve la solicitud de renovación de registro calificado del programa de Odontología de la Universidad del Valle para ser ofrecido bajo la metodología presencial en Cali Valle del Cauca".

17. UNIVERSIDAD DEL VALLE. Resolución 038 del 28 de febrero de 2019 "Por la cual se modifica la Resolución No. 096 del 9 de septiembre de 2010 Consejo Académico que 
define la estructura curricular del Programa Académico de Odontología"

18. MINISTERIO DE EDUCACIÓN NACIONAL. Resolución 8954 del 27 de agosto de 2019 "Por medio de la cual se aclara de oficio y se resuelve la solicitud del registro calificado del programa de Odontología, ofrecido bajo la metodología presencial en Cali (Valle del Cauca)".

19. SUPERINTENDENCIA DE INDUSTRIA Y COMERCIO. Resolución 31716 del 30 de julio de 2019 "Por la cual se otorga una patente de invención".

20. SUPERINTENDENCIA DE INDUSTRIA Y COMERCIO. Resolución 60312 del 5 de noviembre de 2019 "Por la cual se otorga una patente de invención”.
21. SUPERINTENDENCIA DE INDUSTRIA Y COMERCIO. Resolución 60290 del 5 de noviembre de 2019 "Por la cual se otorga una patente de invención".

22. UNIVERSIDAD DEL VALLE. Resolución 113 del 5 de septiembre de 2019 "Por la cual se define la estructura curricular del Programa Académico Especialización en Cirugía Oral y Maxilofacial"

23. UNIVERSIDAD DEL VALLE. Resolución 067 del 14 de noviembre de 2019 "Por la cual se crea el programa académico Especialización en Cirugía Oral y Maxilofacial” 